\title{
Facial onset sensory and motor neuronopathy
}

INSERM

\section{Source}

INSERM. (1999). Orphanet: an online rare disease and orphan drug data base. Facial onset sensory and motor neuronopathy. ORPHA:85162

Facial onset sensory and motor neuronopathy is characterised initially by paraesthesia and numbness in the region of the trigeminal nerve distribution, which later progresses to involve the scalp, neck, upper trunk and upper limbs. Onset of motor manifestations occurs later with cramps, fasciculations, dysphagia, dysarthria, muscle weakness and atrophy. This syndrome has been described in four males and appears to be a slowly progressive neurodegenerative disease. 